${ }^{1,2}$ G A F S van Liere, ${ }^{1,2} \mathrm{C}$ J P A Hoebe, ${ }^{2} \mathrm{P}$ F G Wolffs, ${ }^{1,2}$ N H T M Dukers-Muijers. 'Department of Sexual Health, Infectious Diseases and Environmental Health, Public Health Service South Limburg, Geleen, The Netherlands; ${ }^{2}$ Department of Medical Microbiology, Maastricht University Medical Center, Maastricht, The Netherlands

Introduction Symptom- and sexual history-based testing (testing on indication) for STD in (high risk) women has become part of standard operating procedures in STD clinics. However, little is known about alternative transmission routes for example by fingers and toys. We determined the proportion anorectal STD missed when applying testing on indication, such as STD due to alternative transmission routes.

Methods All women attending our STD clinic (consults: $\mathrm{n}=395$ ) [from May 2012-December 2012] were routinely tested for anorectal and urogenital Chlamydia trachomatis $(\mathrm{Ct})$ and Neisseria gonorrhoeae $(\mathrm{Ng})$ infections. Data were collected on demographics, anal symptoms, anal sex with casual/steady partner(s) and anal use of fingers and/or toys. We compared anorectal STD (Ct and/or Ng) prevalence between 3 groups of women: with indication (self reported anal symptoms and/or anal sex), without indication (no symptoms, no anal sex, no fingers/toys) and without indication but with self reported anal use of fingers/toys. Enrollment in the study is ongoing.

Results In total, 395 consults were included by 380 females. Overall prevalence of anorectal STD was $7.8 \%(n=31)$. Of all consults, $31 \%(n=122)$ had indication for anal testing, 60\% $(n=237)$ no indication and $9 \%(\mathrm{~N}=36)$ only used fingers/toys. Prevalence was $8.2 \%(10 / 122)$ with indication, 8.0\% (19/237, $\mathrm{P}=0.95)$ without indication and $5.6 \%(2 / 36, \mathrm{P}=0.61)$ without indication with use of fingers/toys. Testing on indication only would have missed $68 \%$ of all detected anorectal STD (21/31). Multiple infection (vaginal and anorectal) was present in all but one (with indication).

Discussion Large part of anorectal STD are missed in STD clinics, this was partly associated with alternative transmission routes. Also autoinoculation may be possible, all missed anorectal infections coincided with urogenital STD. However, treating urogenital Ct does not automatically imply appropriate co-treatment of anorectal $\mathrm{Ct}$, since there is current debate on treatment of extragenital $\mathrm{Ct}$.

\section{P3.005 THE BURDEN OF BACTERIAL VAGINOSIS: WOMEN'S EXPERIENCE OF LIVING WITH RECURRENT BACTERIAL VAGINSOSIS}

doi:10.1136/sextrans-2013-051184.0465

1 J E Bilardi, ${ }^{2} \mathrm{~S}$ Walker, ${ }^{2} \mathrm{M}$ Temple-Smith, ${ }^{2 \mathrm{R}} \mathrm{McNair},{ }^{3} \mathrm{~J}$ Mooney-Somers, ${ }^{2} \mathrm{C}$ Bellhouse, ${ }^{4} \mathrm{~S}$ Peterson, ${ }^{5} \mathrm{C}$ K Fairley, ${ }^{5} \mathrm{M}$ Y Chen, ${ }^{5} \mathrm{C}$ Bradshaw. ${ }^{1}$ Monash University, Melbourne, Australia; ${ }^{2}$ The University of Melbourne, Melbourne, Australia; ${ }^{3}$ The University of Sydney, Sydney, Australia; "Melbourne Sexual Health Centre, Melbourne, Australia; ${ }^{5}$ The University of Melbourne \& Melbourne Sexual Health Centre, Melbourne, Australia

Background Bacterial vaginosis (BV) is a common vaginal infection, causing an abnormal vaginal discharge and/or odour in up to $50 \%$ of sufferers. Recurrence is common following recommended treatment. There are limited published data on women's experience of $\mathrm{BV}$, and the impact of recurrent BV on their self-esteem, sexual relationships and quality of life. The aim of this study was to explore the experiences and impact of recurrent BV on women.

Methods Social constructionism informed the epistemological framework of this study. Thirty five women with male and/or female partners were interviewed face-to-face or by phone about their experience of recurrent BV. All interviews were digitally recorded, transcribed verbatim and imported into N-Vivo 9 for thematic analysis

Results Interviews took 20-45 minutes. Median number of diagnosed BV episodes in participants was 3 (range 2-25). Most women attributed BV to some form of sexual contact including specific sexual partners, frequency of sex, unprotected sex or certain sexual practises. The impact of BV varied according to severity of symptoms - the more severe, the greater the impact. The most distressing symptom was abnormal odour, with women commonly feeling embarrassed, ashamed, unattractive, 'dirty' and concerned others may detect the odour. The biggest impact was on women's sex lives, with women commonly avoiding sexual activity, especially oral sex and employing preventative practises to minimise odour including frequent showering and self-help remedies. Women commonly felt confused and frustrated about why they were experiencing recurrent $\mathrm{BV}$, the lack of effective treatment and preventative options and poor public and professional knowledge around BV.

Conclusion Recurrent BV impacted on women broadly and significantly in this study but varied according to symptom severity. Women would like a greater understanding about the cause of BV, better available treatment options and improved knowledge and support amongst clinicians.

\section{P3.006 PREVALENCE OF HIV AND SYPHILIS AMONG VOLUNTARY BLOOD DONORS AT A REGIONAL BLOOD CENTRE IN SRI LANKA FOR THREE YEAR PERIOD}

doi:10.1136/sextrans-2013-051184.0466

L Morawakage. Regional Blood Centre, National Cancer Institute of Sri Lanka, Maharagama, Sri Lanka

Background This study was conducted to estimate the prevalence of HIV and Syphilis among voluntary blood donors at a regional blood centre in western province of Sri Lanka, contributing to $7 \%$ of total blood collection in the country.

All units collected by the blood centre were screened for HIV1 \& 2, HBV, HCV, syphilis and Malaria. $4^{\text {th }}$ generation Enzyme immunoassay (EIA) for HIV p24 antigen and HIV-1 and 2 antibodies (Genscreen-ULTRA HIV Ag-Ab) was the screening test for HIV and confirmation was done by standard immunoblotting (western blot) technique.

Venereal Disease Reference Laboratory (VDRL) test was used for screening of syphilis confirmed by Treponema pallidum hemagglutination (TPHA) test

Method This is a descriptive analysis of retrospective donor records from January 2010 to December 2012.

Results 66087 allogenic donation (Community, apheresis, inhouse) records were analysed. $77.5 \%$ of donors were male and $37.3 \%$ of donors were within 26-35year age group. 91\% of donations were collected from community based donation campaigns.

Overall prevalence of HIV was $0.00004 \%$ ( 3 cases) and incidence was $0.0001 \%, 0.000 \%$ and 0.00004 in 2010, 2011 and 2012 respectively. Overall prevalence of syphilis was $0.0005 \%$ (37 cases) and was $0.0007 \%, 0.0007 \%$ and $0.0006 \%$ in 2010,2011 and 2012 respectively. There were no HIV positive female blood donors and HIV prevalence among male donors was $0.00005 \%$. Prevalence of syphilis in female donors was $0.0002 \%$ and $0.0006 \%$ in male donors. The highest Syphilis prevalence of $0.0007 \%$ was in $26-35$ year age group. All HIV cases were in 36-45 year age group.

Conclusion In 2011, HIV prevalence was $<0.1$ in adult general population of Sri Lanka and reported cases of syphilis was 799. This study shows a low prevalence among blood donors due to the existing strategies of the National blood service which could further improved by strengthening of donor selection and testing strategies.

\section{P3.007* GONORRHOEA, SYPHILIS, CHLAMYDIA AND} TRICHOMONAS IN CHILDREN UNDER THIRTEEN YEARS OF AGE: NATIONAL SURVEILLANCE IN THE UK AND REPUBLIC OF IRELAND 